\title{
Decelerating Neutral Dipolar Molecules
}

\author{
Hendrick L. Bethlem, Giel Berden, and Gerard Meijer \\ Department of Molecular and Laser Physics, University of Nijmegen, \\ Toernooiveld 1, NL-6525 ED Nijmegen, The Netherlands
}

(Received 19 February 1999; revised manuscript received 28 May 1999)

\begin{abstract}
It is experimentally demonstrated that a beam of neutral dipolar molecules can be efficiently decelerated with a time-varying electric field. A pulsed beam of neutral metastable $\mathrm{CO}$ molecules is slowed down from $225 \mathrm{~m} / \mathrm{s}\left(E_{\mathrm{kin}}=59 \mathrm{~cm}^{-1}\right)$ to $98 \mathrm{~m} / \mathrm{s}\left(E_{\mathrm{kin}}=11 \mathrm{~cm}^{-1}\right)$ upon passage through an array of 63 synchronously pulsed electric field stages.

PACS numbers: 33.80.Ps, 33.55.Be
\end{abstract}

Slowing down atoms using laser cooling, together with techniques like magnetic trapping and evaporative cooling, has led to numerous exciting results over the last decade [1], with the realization of Bose-Einstein condensation as the most spectacular achievement [2]. Laser-cooling schemes are applicable to atoms since closed multilevel systems can be realized and many consecutive absorptionemission cycles can be induced. This leads to a significant momentum transfer from the photons of the laser to the atom, resulting in a change of the velocity of the atom.

There currently is a great interest in producing cold molecules for the study of cold molecule-molecule collisions, molecular quantum collective effects, Doppler-free spectroscopy, frequency standards, as well as many other applications already realized for atoms [3]. Slowing down of neutral molecules, a prerequisite for being able to trap them, has proven to be considerably more difficult to be achieved, however. The above-mentioned laser-cooling scheme cannot be applied to slow down molecules as no simple closed-level systems can be found; the unavoidable off-resonant radiative decay of molecules from the excited state to other vibrational levels in the electronic ground state hampers efficient momentum transfer [4].

Once molecules are present at sufficiently low kinetic energies, below a fraction of a $\mathrm{cm}^{-1}$, they can be trapped in various ways. Trapping of neutral molecules in inhomogeneous magnetic [3] and electric fields [5] and trapping based on the use of evanescent fields close to the dielectricvacuum interface have been proposed [6]. It has also been suggested that extremely high laser powers might be used to induce a dipole moment in polarizable molecules, leading to trapping of these molecules in the laser beam [7].

Other methods to produce slow molecules have been put forward and have recently been successfully applied to produce the first samples of trapped molecules. By injecting molecules in a cold buffer gas, sufficiently low kinetic energies of the injected molecules can be obtained after thermalization that they can subsequently be trapped in an inhomogeneous magnetic field. This has recently been demonstrated for $\mathrm{CaH}$ injected in ${ }^{3} \mathrm{He}$ buffer gas at temperatures of around $0.3 \mathrm{~K}$ [8]. Associative collisions between ultracold trapped atoms [9] have proven to be another viable route to create slow molecules [10] which can subsequently be optically trapped [11].

There is yet an alternative method to slow down molecules, based on the interaction of dipolar molecules with electric fields. Molecules possessing an electric dipole moment will gain Stark energy upon entering an electric field when in an appropriate quantum state. This gain in Stark energy ("potential" energy) is compensated by a loss in kinetic energy. If the electric field is switched off before the molecule has left the electric field the molecule will not regain the lost kinetic energy. By letting the molecules pass through multiple pulsed electric fields they can thus be slowed down and brought to a standstill. Straightforward as this approach is, it had up to now never been experimentally demonstrated, even though it has obviously been considered [12-15]. In the late $1950 \mathrm{~s}$ this approach was attempted to decelerate a beam of $\mathrm{NH}_{3}$ molecules in order to produce ammonia maser radiation with a largely reduced linewidth [12]. In the mid 1960s a similar approach was used in order to accelerate a beam of dipolar molecules (LiF, in particular) to superthermal energies [15]. Both attempts have been unsuccessful, and have since then never been actively pursued; the advent of lasers and supersonic seeded nozzle beams made the "Stark-decelerator" and the "Stark-accelerator" approaches for these specific research goals obsolete.

In this Letter we report on the successful implementation of a version of the Stark decelerator to produce a pulsed beam of slow molecules. The electric field geometry is designed such that there is a net focusing effect on the molecular beam, forcing the molecules to stay together throughout the deceleration process. State-selected, metastable CO molecules are slowed down from 225 to $98 \mathrm{~m} / \mathrm{s}$, corresponding to a more than fivefold decrease in kinetic energy.

The experimental setup consists of a compact molecular beam machine, schematically indicated in Fig. 1. An intense pulsed beam of $\mathrm{CO}$ is produced by expanding a $5 \% \mathrm{CO}$ in Xe mixture through a cooled, $1.0 \mathrm{~mm}$ diameter, exit channel mounted onto a pulsed valve (R. M. Jordan Company), into vacuum. Cooling the exit channel to $160 \mathrm{~K}$ brings the mean velocity of the $\mathrm{CO}$ molecules in the 


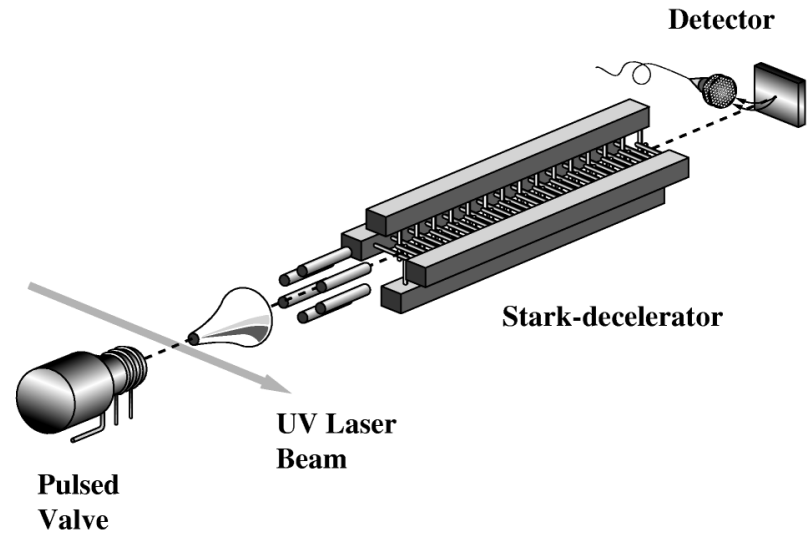

FIG. 1. Schematic of the experimental setup. State-selected metastable CO $\left(a^{3} \Pi_{1}, v^{\prime}=0, J^{\prime}=1\right)$ molecules are prepared using pulsed laser excitation of ground state $\mathrm{CO}$ molecules. The beam of metastable $\mathrm{CO}$ molecules is collimated in a pulsed hexapole, and a subset of these molecules is subsequently slowed down upon passage through a series of 63 pulsed electric field stages. The time-of-flight (TOF) distribution of the metastable $\mathrm{CO}$ molecules over the $71 \mathrm{~cm}$ distance from laser preparation to detection is measured via recording of the amount of electrons emitted from a gold surface when the metastable $\mathrm{CO}$ molecules impinge on it.

"coldest" part of the pulse down to $230 \mathrm{~m} / \mathrm{s}$, corresponding to a kinetic energy of $E_{\mathrm{kin}}=62 \mathrm{~cm}^{-1}$. Preparation of a pulsed beam of metastable $\mathrm{CO}$ molecules in single quantum levels of the first electronically excited $a^{3} \Pi$ state is performed by direct laser excitation of the ground state molecules on the spin-forbidden $a^{3} \Pi\left(v^{\prime}=0\right) \leftarrow$ $X^{1} \Sigma^{+}\left(v^{\prime \prime}=0\right)$ transition (Cameron band), a few $\mathrm{cm}$ downstream from the nozzle orifice. For this, the frequency tripled output of a pulsed dye amplified cw ring dye laser system, delivering spectrally bright tunable pulsed radiation $(1 \mathrm{~mJ}$ in a $5 \mathrm{~ns}$ pulse; spectral width $150 \mathrm{MHz}$ ) around $206 \mathrm{~nm}$, is used. The metastable $\mathrm{CO}\left(a^{3} \Pi\right)$ molecules have a relatively large dipole moment of 1.37 Debye and experience a linear Stark effect when put into an electric field [16]. Consequently, the metastable CO molecules can be (de)focused using spatially inhomogeneous electric fields, while their velocity can be changed in time-varying fields. In our experimental setup, the molecules need to be prepared in states that experience a positive Stark effect (low-field seeking states) which are the upper components of the $\Lambda$ doublets in the $a^{3} \Pi$ state. In the experiments reported here, laser preparation of the $J^{\prime}=1, M \Omega=0,-1$ $a^{3} \Pi_{1}$ level via the $Q_{2}(1)$ transition is used throughout. The radiative lifetime of the metastable $\mathrm{CO}$ molecules in the thus prepared quantum state is $3.7 \mathrm{~ms}$ [17].

After laser preparation, the metastable $\mathrm{CO}$ molecules pass through a $1.0 \mathrm{~mm}$ diameter skimmer into a second, differentially pumped, vacuum chamber and fly into a 5$\mathrm{cm}$-long pulsed hexapole. The inhomogeneous electric field in the hexapole acts as a positive lens for the laser prepared molecules in the $M \Omega=-1$ level [18]. As the metastable molecules are all equally long in the pulsed hexapole field, there is no longitudinal velocity selection in the hexapole, and the effect of the hexapole is that of "transverse cooling" of the beam.

After exiting the hexapole the molecules enter the $35 \mathrm{~cm}$ long Stark decelerator. The decelerator consists of an array of 63 equidistant electric field stages. The electric field in each stage is formed by applying a high voltage to two parallel 3-mm-diameter cylindrical rods centered $4.6 \mathrm{~mm}$ apart, leaving a $1.6 \mathrm{~mm}$ opening for the molecular beam. The two opposing rods are simultaneously switched by two independent high-voltage switches to maximum voltages of +10 and $-10 \mathrm{kV}$, yielding maximum electric fields of $125 \mathrm{kV} / \mathrm{cm}$. With presently available high-voltage switches we can only switch back and forth between \pm 2 and $\pm 10 \mathrm{kV}$, limiting the switching of the electric fields to a maximum of $100 \mathrm{kV} / \mathrm{cm}$. As there is an electric field minimum on the molecular beam axis, there is a net force driving the molecules in low-field seeking states towards this axis. The pairs of rods of adjacent electric field stages are alternately positioned horizontally and vertically to get focusing of the metastable molecules in either direction. All "horizontal" and all "vertical" fields are electrically connected and alternately switched, requiring a total of four independent high-voltage switches (Behlke Electronic GmbH, HTS-150 PGSM). In the present setup, the molecules can lose a maximum of about $0.8 \mathrm{~cm}^{-1}$ kinetic energy per stage [16]. The actual number of electric field stages that is used in the deceleration process can be varied by adjusting the pulse sequence used to trigger the switches. The timing sequence of the pulsed fields is chosen such that the "bunch" of molecules that is intended to be slowed down always has the same phase, i.e., that the bunch of molecules is always centered around the same equilibrium position on the slope of the potential hill when the fields are switched. This is identical to the use of phase stability in synchrotronlike accelerators which provides the opportunity to maintain resonance between a bunch of particles with the interacting fields for indefinite times [19]. Depending on the phase that is chosen, more or less energy is lost (or gained) by the molecules per stage. At the same time the region of stable phase oscillation, and thus the width of the final velocity distribution of the bunch of molecules that is slowed down, is varied. With a total length of the Stark decelerator of only $35 \mathrm{~cm}$, the metastable $\mathrm{CO}$ molecules experience an acceleration in here on the order of $-10^{5} \mathrm{~m} / \mathrm{s}^{2}$, comparable to what can be obtained in laser cooling of atomic beams.

The velocity distribution of the metastable $\mathrm{CO}$ molecules exiting the Stark decelerator is determined by directly recording their flight time through the apparatus. For this, the electrons that are emitted when the metastable $\mathrm{CO}$ molecules impinge on a flat gold surface positioned $28 \mathrm{~cm}$ downstream from the exit of the Stark decelerator are detected as a function of the time since laser preparation of these molecules. The detection efficiency is assumed to 
be independent of the velocity of the impacting metastable $\mathrm{CO}$ molecules, and the absolute detection efficiency of this method for metastable $\mathrm{CO}(6 \mathrm{eV}$ internal energy) is in the $10^{-3}-10^{-2}$ range.

In Fig. 2 a series of measured time-of-flight (TOF) distributions of laser prepared metastable $\mathrm{CO}$ is shown for an increasing number of electric field stages used in the deceleration process, as indicated in the figure. The lower curve is the TOF profile when only the pulsed hexapole is used. As the total distance between the point of laser preparation and detection is $71 \mathrm{~cm}$, this TOF profile corresponds to an original velocity distribution centered around $230 \mathrm{~m} / \mathrm{s}$ with a FWHM of $45 \mathrm{~m} / \mathrm{s}$. With the number of electric field stages increasing, a change in both intensity and shape is observed in the TOF distributions due to the combined effects of focusing and velocity changes. It should be noted that there is no longer a one-to-one correspondence

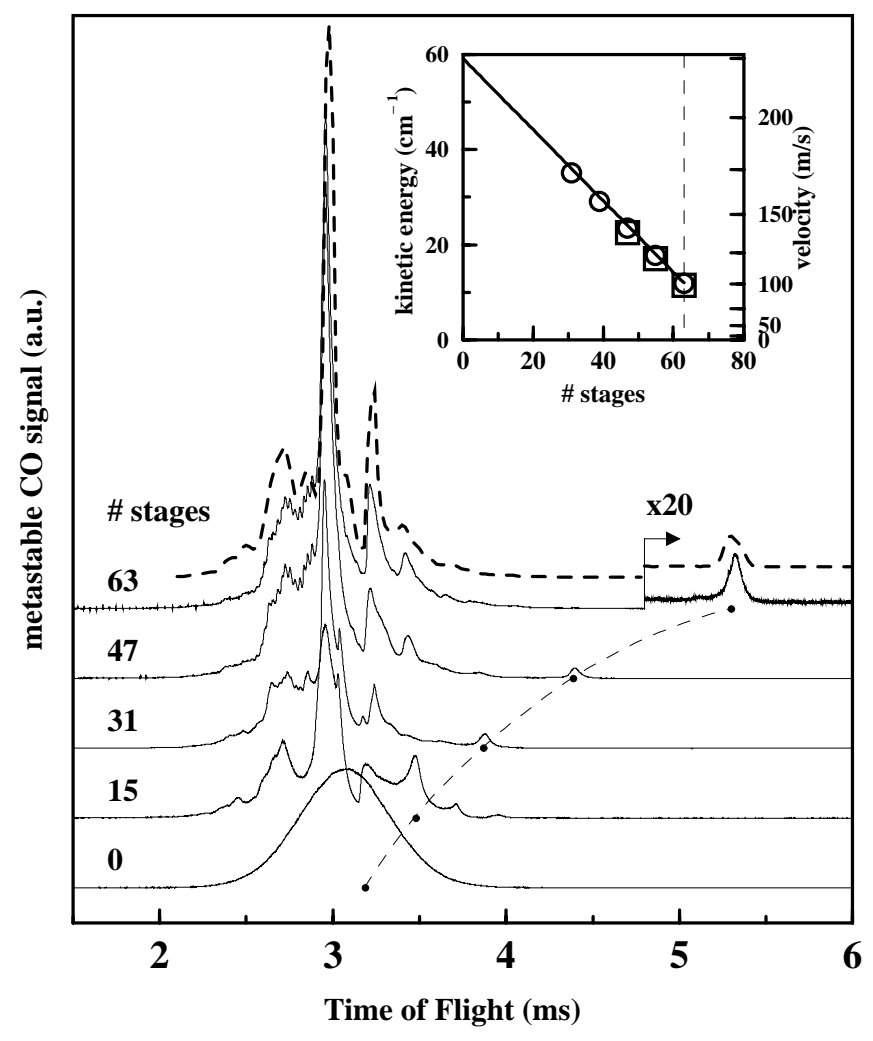

FIG. 2. Series of TOF distributions of metastable CO molecules over the $71 \mathrm{~cm}$ path length through the apparatus. An increasing number of electric field stages has been used. The measured curves are given an offset for clarity, but are all on the same vertical scale. The dashed curve shows a calculated TOF profile, to be compared to the upper of the measured curves. The dots on the baseline of the various distributions, connected by the thin dashed line, indicate the expected arrival time of the decelerated molecules. In the inset the kinetic energy (velocity) for the decelerated molecules, using either the measured (squares) or calculated (circles) time at which the molecules exit the decelerator, is given, together with the expected curve (solid line) for our specific experimental geometry. between the measured TOF profiles and the final velocity distributions when the velocity is changed upon passage of the molecules through the series of pulsed electric fields.

For the measurements presented in Fig. 2, the timing sequence is set such as to slow down molecules that enter the Stark decelerator with a central velocity of $225 \mathrm{~m} / \mathrm{s}$. With the phase set such that the molecules lose on average $0.75 \mathrm{~cm}^{-1}$ of kinetic energy per stage, a bunch of molecules with a FWHM velocity distribution of $4 \mathrm{~m} / \mathrm{s}$ centered around this central velocity is captured. As most of the molecules have initial velocities outside this selected interval, their velocity is, on average, not changed in this process and they only contribute to the intense, structured distribution centered around the original arrival time in the TOF profiles.

With an increasing number of electric field stages a subset of molecules is seen to arrive at an ever later time at the detector. This subset of molecules (on the order of $1 \%$ of the total number of molecules exiting the decelerator) is forced to stay together as a compact bunch in the deceleration process.

With the experimental beam geometry, including the high-voltage pulse sequence applied, and the known Stark energy of metastable $\mathrm{CO}$ in the selected quantum state as input, calculations have been performed to simulate the experimental observations. For the upper curve, in which the maximum number of electric field stages is employed, the calculated TOF profile is shown as well (dashed curve) and is seen to describe the experimental observations very well. Although not shown explicitly, all calculated TOF profiles are in good agreement with the observed ones, and both the observed intensity changes with an increasing number of electric field stages as well as the rather complicated structures that appear in the TOF profiles are well reproduced. The dots on the baseline of the various distributions, connected by the thin dashed line, indicate the expected arrival time of the decelerated molecules.

For the subset of molecules that is slowed down, it is known from the timing sequence when they exit the Stark decelerator, and their velocity can thus be calculated from their flight time over the known distance from the last electric field stage to the detector. As an independent check, the time at which the bunch of slow molecules exits the Stark decelerator is determined using a second dye laser system in combination with a compact TOF mass spectrometer unit positioned near the exit of the decelerator. Using $(1+1)$-resonance enhanced multiphoton ionization detection of the metastable $\mathrm{CO}$ molecules, with the $b^{3} \Sigma^{+} \leftarrow a^{3} \Pi$ transition around $283 \mathrm{~nm}$ as the resonant step, the calculated timing is experimentally verified. Using either the measured or the calculated timing, the kinetic energy (velocity) of the decelerated molecules as a function of the number of electric field stages is given in the inset of Fig. 2 as squares or circles, respectively. The solid line corresponds to an energy loss of $0.75 \mathrm{~cm}^{-1}$ per stage, and is seen to describe the experimental points very well. 
It should be noted that, different from laser-cooling schemes, it is not possible to increase the phase-space densities of the molecules when time-varying potentials are employed [20]. In the Stark decelerator one basically selects a part out of the original velocity distribution and shifts this as a whole to lower absolute velocities. By choosing the appropriate electrode configuration of the Stark decelerator, its "acceptance angle" is optimized, resulting in a "transmittance" of $\sim 10 \%$ for the molecules with the correct initial velocity. The accepted molecules are then decelerated without decrease in phase-space density. By using an intense pulsed, supersonic molecular beam, very high initial phase-space densities of neutral molecules can be made. The combination of the Stark decelerator with a pulsed molecular beam, therefore, yields unique opportunities to produce slow beams of neutral dipolar molecules.

Our calculations indicate no intrinsic limitation to slowing down the molecules to a standstill without further losses. Metastable CO molecules with velocities below $25 \mathrm{~m} / \mathrm{s}$ can subsequently be trapped in an electric quadrupole trap that is rapidly switched on when the molecules fly through it. Dense clouds of metastable CO molecules (on the order of $10^{7}$ molecules) can thus be produced opening up the possibility for studies of the as yet unexplored physics of the mutually interacting dipolar molecules in these samples.

The main advantage of using metastable $\mathrm{CO}$ for these proof-of-principle experiments is that their full TOF distributions can be readily recorded, which is crucial for experimental verification and optimization of the performance characteristics of the decelerator. Instead of using this scheme on metastable CO in the $\Omega=1$ multiplet we can also laser prepare $\mathrm{CO}$ in the $\Omega=2$ multiplet, which has a substantially longer lifetime of up to $200 \mathrm{~ms}$ [17]. More importantly, the deceleration scheme presented here can be used on a large variety of ground state molecules as well. For this, the molecule under study must have a sufficiently large positive Stark shift $\left(\sim 1 \mathrm{~cm}^{-1}\right)$, either linear or higher order, in experimentally feasible electric fields $(\sim 200 \mathrm{kV} / \mathrm{cm})$. Furthermore, an intense beam with sufficiently low initial kinetic energy is desired. The number of stages that is required to bring the molecules to a standstill solely depends on the ratio of the initial kinetic energy to the obtainable Stark shift. On the basis of these criteria, molecules like $\mathrm{OH}$ and $\mathrm{NH}_{3}$ are excellent candidates and could be stopped in the existing experimental setup, but probably even more interesting is the possibility to perform these experiments with $\mathrm{H}_{2} \mathrm{O}$ molecules. Slow molecular beams of any of these molecules yield new possibilities in molecular beam (surface) scattering and molecular beam reaction experiments in general, apart from offering the possibility to finally trap these species.

This work is part of the research program of the "Stichting voor Fundamenteel Onderzoek der Materie (FOM)," which is financially supported by the "Nederlandse Organisatie voor Wetenschappelijk Onderzoek (NWO)." We acknowledge the expert technical assistance of Ing. A. van Roij and Ch. Timmer, as well as the assistance of Dr. G. C. Groenenboom in setting up the numerical simulations.

[1] S. Chu, Rev. Mod. Phys. 70, 685 (1998); C. N. CohenTannoudji, ibid. 70, 707 (1998); W. D. Phillips, ibid. 70, 721 (1998).

[2] M.H. Anderson, J.R. Ensher, M.R. Matthews, C.E. Wieman, and E. A. Cornell, Science 269, 198 (1995).

[3] B. Friedrich, R. deCarvalho, J. Kim, D. Patterson, J. D. Weinstein, and J. M. Doyle, J. Chem. Soc. Faraday Trans. 94, 1783 (1998).

[4] J. T. Bahns, W. C. Stwalley, and P.L. Gould, J. Chem. Phys. 104, 9689 (1996).

[5] W.H. Wing, Phys. Rev. Lett. 45, 631 (1980).

[6] R. J. Cook and R. K. Hill, Opt. Commun. 43, 258 (1982).

[7] B. Friedrich and D. Herschbach, Phys. Rev. Lett. 74, 4623 (1995).

[8] J. D. Weinstein, R. deCarvalho, T. Guillet, B. Friedrich, and J. Doyle, Nature (London) 395, 148 (1998).

[9] Y.B. Band and P.S. Julienne, Phys. Rev. A 51, R4317 (1995).

[10] A. N. Nikolov, E. E. Eyler, X. T. Wang, J. Li, H. Wang, W. C. Stwalley, and P. L. Gould, Phys. Rev. Lett. 82, 703 (1999).

[11] T. Takekoshi, B. M. Patterson, and R. J. Kinze, Phys. Rev. Lett. 81, 5105 (1998).

[12] J. G. King, in Proceedings of the 13th Annual Symposium on Frequency Control, Asbury Park, 1959 (U.S. Army Signal Research and Development Laboratory, Fort Monmouth, 1959), p. 603.

[13] V.S. Kazachok, Sov. Phys. Tech. Phys. 10, 882 (1965).

[14] D. Auerbach, E.E. A. Bromberg, and L. Wharton, J. Chem. Phys. 45, 2160 (1966).

[15] R. Wolfgang, Sci. Am. 219, No. 4, 44 (1968).

[16] R. T. Jongma, G. von Helden, G. Berden, and G. Meijer, Chem. Phys. Lett. 270, 304 (1997).

[17] R. T. Jongma, G. Berden, and G. Meijer, J. Chem. Phys. 107, 7034 (1997).

[18] R. T. Jongma, Th. Rasing, and G. Meijer, J. Chem. Phys. 102, 1925 (1995).

[19] E. M. McMillan, Phys. Rev. 68, 143 (1945).

[20] W. Ketterle and D. E. Pritchard, Phys. Rev. A 46, 4051 (1992). 